\title{
Estimation and simulation of foraging trips in land-based marine predators
}

\author{
Théo Michelot, ${ }^{1,9}$ Roland Langrock, ${ }^{2}$ Sophie Bestley, ${ }^{3,4}$ Ian D. Jonsen, ${ }^{5}$ \\ Theoni Photopoulou, ${ }^{6,7}$ and Toby A. Patterson ${ }^{8}$ \\ ${ }^{1}$ University of Sheffield, Sheffield, UK \\ ${ }^{2}$ Bielefeld University, Bielefeld, Germany \\ ${ }^{3}$ Australian Antarctic Division, Department of Environment, Kingston, Tasmania, Australia \\ ${ }^{4}$ Institute for Marine and Antarctic Studies, Hobart, Tasmania, Australia \\ ${ }^{5}$ Macquarie University, Sydney, New South Wales, Australia \\ ${ }^{6}$ Nelson Mandela Metropolitan University, Port Elizabeth, South Africa \\ ${ }^{7}$ University of Cape Town, Rondebosch, South Africa \\ ${ }^{8}$ CSIRO Oceans and Atmosphere, Hobart, Tasmania, Australia
}

\begin{abstract}
The behavior of colony-based marine predators is the focus of much research globally. Large telemetry and tracking data sets have been collected for this group of animals, and are accompanied by many empirical studies that seek to segment tracks in some useful way, as well as theoretical studies of optimal foraging strategies. However, relatively few studies have detailed statistical methods for inferring behaviors in central place foraging trips. In this paper we describe an approach based on hidden Markov models, which splits foraging trips into segments labeled as "outbound", "search", "forage", and "inbound". By structuring the hidden Markov model transition matrix appropriately, the model naturally handles the sequence of behaviors within a foraging trip. Additionally, by structuring the model in this way, we are able to develop realistic simulations from the fitted model. We demonstrate our approach on data from southern elephant seals (Mirounga leonina) tagged on Kerguelen Island in the Southern Ocean. We discuss the differences between our 4-state model and the widely used 2-state model, and the advantages and disadvantages of employing a more complex model.
\end{abstract}

Key words: animal telemetry; central place foraging; hidden Markov model; Mirounga leonina; southern elephant seal.

\section{INTRODUCTION}

Central place foraging (CPF) is a widely applied concept in ecology (Olsson and Bolin 2014, Higginson and Houston 2015). Many terrestrial species with home ranges, or shelters, can be regarded as central place foragers (Bell 1990). Colony-based marine animals, such as seabirds and seals, that must moult, breed and raise young on land or ice can also use CPF strategies. It has been hypothesized that, due to density dependence, waters near to the colony may become depleted of prey (Ashmole 1963), or simply that the most profitable prey are spatially separated from land-based colonies, necessitating trips to more distant foraging areas (Oppel et al. 2015). In many seabirds, during phases when the young are being fed and reared, adult birds are constrained to forage within closer range of the colony (Boyd et al. 2014, Patrick et al. 2014). Pinnipeds must also haul out to periodically moult, as well as for mating and rearing young (Russell et al. 2013). These animals are the subject of considerable ongoing research, often utilizing

Manuscript received 13 March 2017; accepted 18 April 2017. Corresponding Editor: Jeffrey S. Shima.

${ }^{9}$ E-mail: tmichelot1@sheffield.ac.uk tracking techniques to collect movement data at sea (Hays et al. 2016). Moreover, many are the subject of intense conservation efforts (Lonergan et al. 2007, Croxall et al. 2012, Hamer et al. 2013, Martin and Crawford 2015, Jabour et al. 2016).

Often, studies aim to assess how CPF animals apportion their time between searching and active foraging, and to identify particular characteristics, for example relating to habitat usage, trip length, activity budgets, and other variables (e.g., Staniland et al. 2007, Raymond et al. 2015, Hindell et al. 2016, Patterson et al. 2016). How these quantities vary with ontogenetic stage or age is often important; for example, naive young animals versus experienced adults, or sex-specific foraging strategies (e.g. Breed et al. 2009, Hindell et al. 2016). To evaluate hypotheses about movements in CPF, it is very helpful to have models which objectively classify movement into different modes (or "phases"), with different statistical properties, indicating differences in underlying behavior (Langrock et al. 2012).

Several recent CPF studies have looked at switching models which include latent behavioral states, but usually these are general models that are not specifically designed to capture the behavioural cycle of CPF (e.g., Breed et al. 2009, Jonsen et al. 2013, Jonsen 2016). 
Latent states are often given labels such as "transit" and "resident". In the latter, the notion of area-restricted search (Kareiva and Odell 1987, Fauchald and Tveraa 2003, Morales et al. 2004) is often invoked. In an area with high foraging returns, the animal is expected to undertake less directed movements, and therefore higher turning rates, and generally lower speeds of travel. These concepts have proven useful for modeling movements of $\mathrm{CPF}$, but explicitly incorporating the trip structure in the model would give a more nuanced understanding of the likely behavioural sequences. At the most basic level, an animal must leave a colony, transit out to (one or more) foraging grounds, search and obtain food, and then eventually return.

Our aim here is to construct a model which captures the following sequence of movement modes: outbound $\rightarrow$ search $\rightarrow$ forage $\rightarrow \ldots \rightarrow$ search $\rightarrow$ inbound. We outline the random walk models which can be used to represent these movement modes, and then show how these can be integrated into the hidden Markov model (HMM) framework. HMMs have been used widely in animal movement modeling (Langrock et al. 2014, McKellar et al. 2015, Auger-Méthé et al. 2016, DeRuiter et al. 2017, Leos-Barajas et al. 2017). They represent a computationally efficient approach for fitting models with discrete latent states to time series data. A thorough description of HMMs for animal movement is given in Langrock et al. (2012).

An important subsequent focus of the model we seek to construct is that it should be suitable for simulation of foraging trips. Simulation is already used in habitat modelling of central place foragers as null models for distribution that account for some areas being inaccessible due to distance constraints (Wakefield et al. 2009, Raymond et al. 2015). However, estimation of such simulation models is often ad-hoc (Matthiopoulos 2003). Beyond the specific case of habitat modelling, even simulation models which capture only a few aspects of foraging behaviour would be useful in making predictions, with associated uncertainty, from finite samples of individuals drawn from populations. The desire to simulate from the fitted model places a requirement of greater realism on the movement model structure. This further motivates the development of the trip-based movement model for CPF.

We acknowledge from the outset that the model we present below is a simplification of the true processes under investigation. Despite the broader applicability of the CPF concept, we restrict our usage to the situation of marine predators that are constrained to return to land after a certain period of time. It should be noted however that the methods we present may well have broader application. CPF is likely to be influenced by patchiness operating at scales which are not observable from the telemetry data, and dependent on local conditions (only some of which may be observable via remote sensing). To fully generalize our methods, inclusion of environmental data will be necessary (Labrousse et al. 2015).
Considerations of these ideas have tended to employ computationally demanding techniques (e.g., McClintock et al. 2012), which can limit their applicability with large data sets. So far, models for large data sets (i.e., many observed locations) have been underrepresented.

Herein, we describe the modeling framework and demonstrate estimation using data from southern elephant seals (Mirounga leonina). We then show simulation of foraging trips from the fitted model, and assess which aspects of the real trips are replicated well and which are not. Finally, we discuss how a model of this type can be tailored to a given species' case and extended to include other covariates (e.g., environmental) that may influence movement behaviour. Additionally, we discuss the limitations of our model, and how these relate to the general problem of simulation and subsequent prediction of behaviour from fitted multistate movement models.

\section{Methods}

\section{Building blocks for the overall model}

We first describe the types of random walks required to describe the different segments of a foraging trip, and then how these are combined within the full model, an HMM. Fig. 1 illustrates the typical pattern of an elephant seal's foraging trip. The maps were produced with the R package marmap (Pante and Simon-Bouhet 2013).

The trip consists of (at least) three clearly distinct phases of movement: the fast and directed trip from the colony to the sea ice region, a period of slower and less directed movement near the ice, and the fast and directed trip from the ice to the colony. Marine prey resources are patchily (non-uniformly) distributed at multiple spatial scales (Fauchald and Tveraa 2006), so animals will typically still need to search between dynamic favorable forage patches within and around the sea ice and Antarctic shelf regions (see for example inset panels of figure 2 in Bestley et al. 2013). This search may manifest as slower, less directed movement than the migration transits, but faster and more directed movement than area restricted search.

We choose the following four movement modes for our analysis, for the distinct phases within a trip: (1) outbound trip, away from the central place (here, the colony at Kerguelen Island) and towards the foraging regions, characterized by very fast and highly directed movement; (2) search-type movement in the foraging regions, characterized by moderately fast movement and some directional persistence (though without a clear destination); (3) foraging activities, characterized by non-directed, slow movement; and (4) return trip, back to the central place, with very fast and again highly directed movement. We label the movement modes for convenience, but note that each phase of the trip (in particular "search" and "forage") can potentially encompass several behaviours, perhaps even behaviours that are functionally equivalent, 


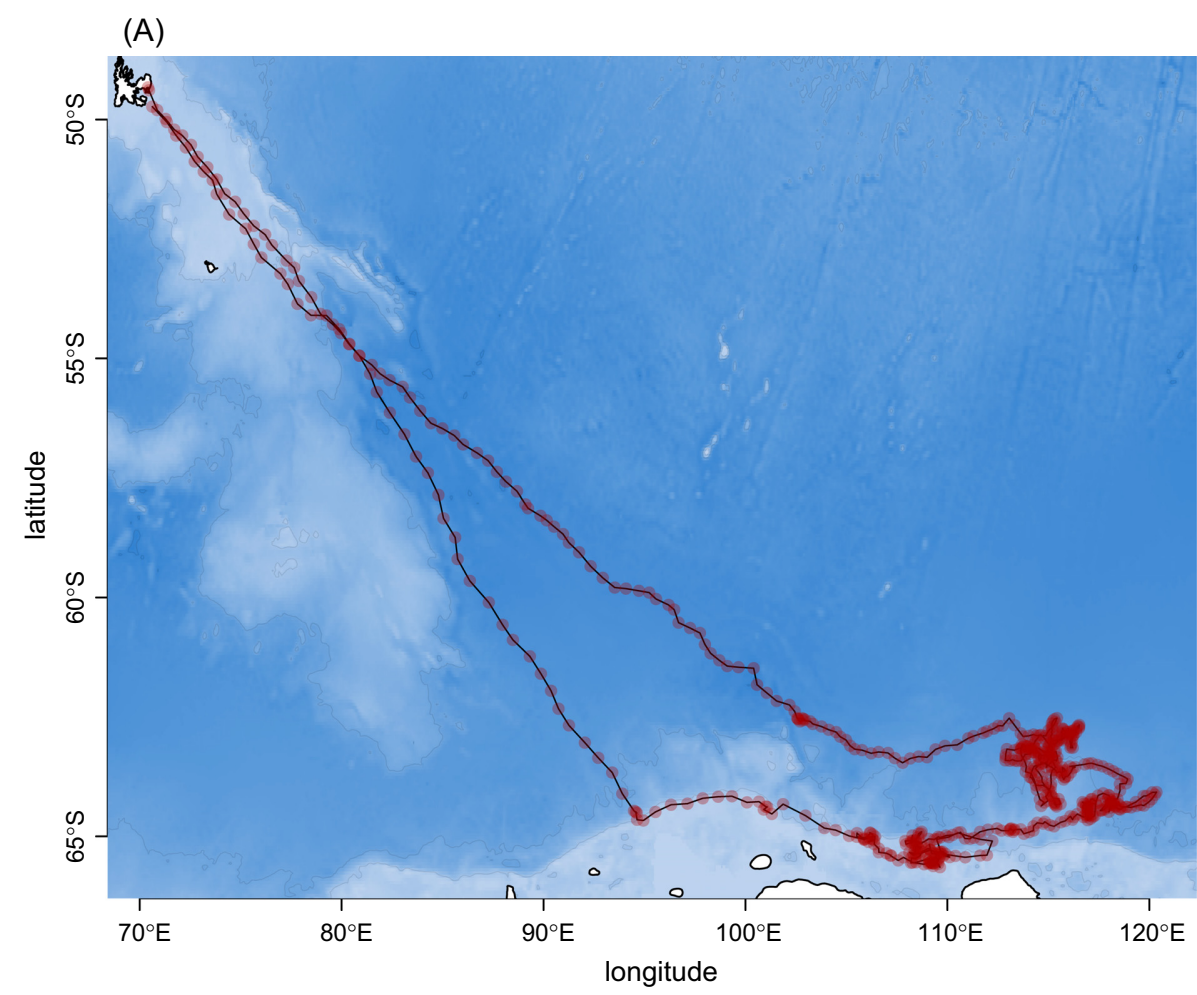

(B)

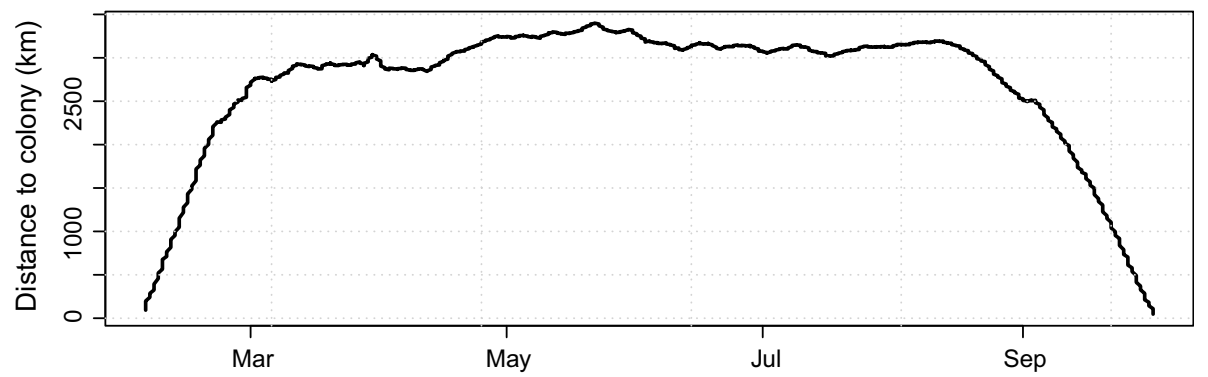

FIG. 1. (A) An example track of a southern elephant seal tagged at Kerguelen Island (top left), which makes an outward foraging trip to the Antarctic continental shelf before returning. (B) The distance from Kerguelen Island through time, clearly showing rapid outward transit, foraging movements over an extended period of time, and the rapid return transit. [Color figure can be viewed at wileyonlinelibrary.com]

like different types of foraging behaviour, as long as they lead to similar movement patterns.

Both (2) and (3) can be adequately modelled using commonly applied correlated random walks (CRWs). CRWs involve correlation in directionality, and can be represented by modelling the turning angles of an animal's track using a circular distribution with mass centred either on zero (for positive correlation) or on $\pi$ (for negative correlation). For example, we could model phase (2), which involves positive correlation in directionality, by assuming

$$
\begin{aligned}
\text { bearing }_{t} \sim \text { von Mises }(\text { mean } & =\text { bearing }_{t-1}, \\
\text { concentration } & =\kappa),
\end{aligned}
$$

with $\kappa$ denoting a parameter to be estimated. (Larger $\kappa$ values lead to lower variance of the von Mises distribution, and hence higher persistence in direction.) Alternatively, the mean can be specified to be bearing $\sin _{t-1}-\pi$, corresponding to an expected reversal in direction, which is often found in encamped, foraging, or resting modes (e.g., "encamped" behaviour in Morales et al. 2004, and "area-restricted search" in Towner et al. 2016). Instead of specifying the mean a priori, it can also be estimated from the data. That is, we can consider

$$
\begin{aligned}
\text { bearing }_{t} \sim \text { von Mises }(\text { mean } & =\text { bearing }_{t-1}+\lambda, \\
\text { concentration } & =\kappa),
\end{aligned}
$$


where $\lambda \in[-\pi, \pi)$ is a parameter to estimate. We note that CRWs can alternatively be constructed by modelling turning angles rather than bearings, leading to equivalent model formulations, with

$$
\text { angle }_{t} \sim \text { von } \operatorname{Mises}(\text { mean }=\lambda, \text { concentration }=\kappa) \text {. }
$$

Choosing between these two formulations is only a matter of convenience.

For modelling phases (1) and (4), biased random walks (BRWs) are better suited. Bias in random walks usually (though not necessarily) refers to a tendency towards (or away from) a particular location, sometimes called a centre of attraction (or point of repulsion). For example, a bias towards a location is obtained by assuming that

$\operatorname{bearing}_{t} \sim \operatorname{von} \operatorname{Mises}\left(\right.$ mean $=\phi_{t}$, concentration $\left.=\kappa\right)$,

where $\phi_{t}=\arctan \left[\left(y_{c}-y_{t}\right) /\left(x_{c}-x_{t}\right)\right]$ is the direction of the vector pointing from the animal's current position, $\left(x_{t}, y_{t}\right)$, to the central place, $\left(x_{c}, y_{c}\right)$.

In principle, it is also possible to construct random walks that are both biased and correlated (BCRWs), thereby trading off directional persistence and possible turning towards the destination. However, in our experience, it is difficult to statistically distinguish CRWs and BRWs. As a consequence, usage of BCRWs tends to lead to numerical instability within the model fitting procedure, as it is challenging to estimate the respective weights of the biased and correlated components of the process. For more details on CRWs, BRWs and BCRWs, see Codling et al. (2008).

As can be seen in Fig. 1, the animal tends to remain within each phase of movement for some time before switching to a different phase. To accommodate both the persistence and the stochastic switching between different phases of the movement, we use an underlying (unobserved) state process. The state process, $S_{1} \ldots, S_{T}$, takes values in $\{1, \ldots, N\}$, such that, at each time $t$, the movement observed follows one of $N$ types of random walks (possibly correlated and/or biased), as determined by the current state. We take the state process to be a Markov chain, which together with the observation process, i.e., the BRWs and CRWs conditional on the current state, defines a hidden Markov model for the animal's movement (HMM; see Chapter 18 in Zucchini et al. 2016). The state process is characterized by the transition probabilities

$$
\gamma_{i j}=\operatorname{Pr}\left(S_{t}=j \mid S_{t-1}=i\right), \text { for } i, j \in\{1, \ldots, N\} .
$$

In the trip-based movement detailed previously, there are $N=4$ states, corresponding to the four phases of movement. Note that, in general, there is no guarantee of a one-to-one equivalence between the (data-driven) states of the Markov chain and the actual behaviours of the animal (Patterson et al. 2016). As such, the states should be interpreted with care, but they are often useful proxies for the behavioural modes. In subsequent sections, we use the terms "behaviours" and "states" interchangeably.

Framing the model as an HMM makes it possible to use the very efficient HMM machinery to conduct statistical inference. In particular, the model parameters can relatively easily be estimated by numerically maximizing the likelihood, which can be calculated using a recursive scheme called the forward algorithm (Zucchini et al. 2016). The main challenge herein lies in identifying the global rather than a local maximum of the likelihood the same or similar problems arise when using the expectation-maximization algorithm or Markov chain Monte Carlo sampling to estimate parameters. The efficiency of the forward algorithm is one of the key reasons for the popularity and widespread use of HMMs, and similar algorithms can be applied for forecasting, state decoding, and model checking. In particular, we use the Viterbi algorithm to decode the most likely sequence of underlying states in section Estimated state sequences. More details on inference in HMMs are given in Zucchini et al. (2016) and, for the particular case of animal movement modelling, in Patterson et al. (2016). For our case study, we used the R optimization function $\mathrm{n}$ Im to numerically maximize the likelihood, which was partly written in $\mathrm{C}++$ for computational speed.

\section{Case study data}

The southern elephant seal (Mirounga leonina) is a pinniped top predator with a circumpolar distribution throughout high latitudes in the southern hemisphere (Carrick et al. 1962). Haul out phases on land occur at fairly predictable times during the annual cycle for moulting and breeding (Hindell and Burton 1988). The species is known for making large scale migrations from isolated subantarctic land colonies, both southwards to the sea ice zone around the Antarctic continental margin, and also into open ocean pelagic zones (Biuw et al. 2007, Labrousse et al. 2015, Hindell et al. 2016). A recent study (Hindell et al. 2016) applied two-state movement models (sensu Morales et al. 2004) to a large data set of several hundred individual tracks. This study was focused on detecting basin-scale patterns in foraging effort, rather than explicitly modelling the sequence of behaviours within individual foraging trips.

In this case study, we examine trips from 15 animals (eight adult females and seven subadult males) tagged at Kerguelen Island. These animals were fitted with telemetry units of the Sea Mammal Research Unit (St Andrews, UK) which transmit data via the Argos satellite network (Photopoulou et al. 2015). Frequently, the elephant seals remained at the colony for extended periods at the start of the time series, and similarly after returning from foraging trips. Because of the HMM structure, the highly stationary data from these periods holds little information and rather serves to leave 
potential for numerical instability. Data from these periods were removed prior to use in the HMM. Near stationary periods immediately prior to colony departure and/or following return to the colony were identified for removal by examining one-dimensional time series plots of the Argos longitude and latitude observations. The truncated trips lasted between five and eight months, except for two incomplete trips, during which the data collection was interrupted before the animals returned to the colony (tags may fail before trip completion).

Due to the occasional large errors and the irregular timing of the Argos location observations, these data were filtered using a state-space model (SSM, Jonsen et al. 2013) to obtain a regular time sequence of location estimates with reduced uncertainty. The SSM used was a variant of that described in Jonsen et al. (2005), implemented with the $\mathrm{R}$ package TMB (Kristensen et al. 2016). Associated R and C++ code, for pre-processing the Argos data, are available on Github, at github.com/ ianjonsen/ssmTMB. The state-space model was fit with a 2.4-h time step, yielding ten location estimates per day. Other time steps were evaluated but $2.4 \mathrm{~h}$ provided the best fit according to AIC and auto-correlation functions of the residuals. This time step resulted in calculated step lengths (speeds) and turning angles (or, equivalently, bearings) that had relatively low contrast between movement phases apparent in the observed data. Accordingly, we sub-sampled the estimated locations to every fourth time step (i.e. $9.6 \mathrm{~h}$ frequency). The data provided to the HMM were step lengths and turning angles, as described in section Building blocks for the overall model.

\section{Model details}

For the elephant seal case study, we employ the structure of a four-state HMM as described in section Building blocks for the overall model. The first state corresponds to the outbound trip from the colony to a foraging region, and is modelled by a BRW with repulsion from the colony. The animal then alternates between states 2 ("search") and 3 ("forage"), each using a CRW. Finally, the process switches to the inbound trip, modelled by a BRW with attraction towards the colony. The movement is measured in terms of step lengths and turning angles - modelling the latter is equivalent to modelling bearings, but here easier to implement. We use a gamma distribution to model the step lengths in each state, and a von Mises distribution for the turning angles. The mean turning angles are estimated for both statedependent CRWs (in states 2 and 3, respectively), instead of being fixed a priori. This results in fourteen parameters to estimate at the level of the observation process: four shape and four scale parameters (for the gammadistributed steps), plus four concentration parameters and two means (for the angles). For states 1 and 4, no mean parameter needs to be estimated for the associated $\mathrm{BRW}$, as the expected direction is determined through the bias.
Using the notation introduced in section Building blocks for the overall model, we write the transition probability matrix as

$$
\Gamma=\left(\begin{array}{cccc}
\gamma_{11} & \gamma_{12} & 0 & 0 \\
0 & \gamma_{22} & \gamma_{23} & \gamma_{24} \\
0 & \gamma_{32} & \gamma_{33} & 0 \\
0 & 0 & 0 & 1
\end{array}\right)
$$

This structure ensures that the sequence of states follows the behavioural cycle described in the Introduction, by preventing some transitions. Note that we could choose $\gamma_{13}$ and $\gamma_{34}$ to be non-zero, to allow the process to switch from outbound to forage, and forage to inbound, respectively. In this case study, we decided to prevent these transitions, i.e., we assumed a transitional regime of moderately fast and directed movement (search) between a phase of fast and directed movement (outbound or inbound), and a phase of slow non-directed movement (forage).

This formulation also leads to improved numerical stability of the estimation, as it reduced the number of parameters to estimate. Here, state 4 is an absorbing state, as we only consider tracks comprising (at most) one trip away from the colony. It would be straightforward to relax this constraint, e.g., by choosing $\gamma_{41}>0$. For tracks comprising several years of data, a fifth state could be added to model the movement of the seals at the colony.

In analyses like this one, it is often of interest to understand the drivers of behavioural switches, by expressing the transition probabilities as functions of time-varying covariates (see, e.g., McKellar et al. 2015, Breed et al. 2016). Here, we introduce two covariates: the great-circle distance to the colony from the location at time $t, d_{t}$, and the time since departure from the colony, $t-t_{0}$. These are included for two key aspects which are both relevant for CPF foraging behaviour and are also necessary to construct a model which will replicate trips in a simulation setting. Specifically, we need to model the fact that animals often make fast directed trips away from the colony and tend to switch into other movement modes once they have reached foraging grounds. In this case we use distance from the colony as a covariate affecting the probability of transitioning from outbound to search,

$$
\gamma_{12}^{(t)}=\operatorname{logit}^{-1}\left(\beta_{0}^{(12)}+\beta_{1}^{(12)} d_{t}\right) .
$$

Somewhat similarly, animals cannot remain at sea indefinitely. Therefore, we use time since departure from the colony as a covariate on the switch from search to inbound $\left(\gamma_{24}\right)$. As there are two non-zero probabilities of switching out of state 2, we use a special case of the multinomial logit link, the general expression of which is given e.g., in Patterson et al. (2016). In our model, 


$$
\begin{gathered}
\gamma_{23}^{(t)}=\frac{\exp \left(\beta_{0}^{(23)}\right)}{1+\exp \left(\beta_{0}^{(23)}\right)+\exp \left(\eta_{24}\right)}, \text { and } \\
\gamma_{24}^{(t)}=\frac{\exp \left(\eta_{24}\right)}{1+\exp \left(\beta_{0}^{(23)}\right)+\exp \left(\eta_{24}\right)},
\end{gathered}
$$

where

$$
\eta_{24}=\beta_{0}^{(24)}+\beta_{1}^{(24)}\left(t-t_{0}\right)
$$

The $\beta_{k}^{(i j)} \in \mathbb{R}$ are parameters to be estimated. Note that, because the rows of the transition probability matrix must sum to $1, \gamma_{11}$ and $\gamma_{22}$ are also time-varying in this example. There are six parameters to estimate in the state-switching process: the five $\beta_{k}^{(i j)}$ coefficients and $\gamma_{32}$. The remaining elements of the matrix, i.e., $\gamma_{11}, \gamma_{22}$ and $\gamma_{33}$, are obtained from the row constraints. This results in a total of twenty parameters to estimate: fourteen parameters for the state-dependent distributions of steps and angles, and six parameters for the transition probabilities.

\section{Simulation from fitted model}

Having estimated model parameters from the real data, it is possible to simulate movement from the model described in section Model details. A simulated track starts near Kerguelen Island, in state 1 (outbound trip). The bearing is initialized from a von Mises distribution with a mean pointing towards the South, to mimic the elephant seals' movement. The directionality of the movement in state 1 ensures that the trajectory goes southward, overall. At each time step, the state process is simulated from the estimated (possibly time-varying) switching probabilities. Then, a step length and a bearing are simulated from the estimated gamma and von Mises distributions, respectively. The corresponding longitude and latitude coordinates are derived using the $\mathrm{R}$ package geosphere (Hijmans 2016). The new location is rejected if it is on land, using the borders defined in the data set wr ld_simpl of the maptools $\mathrm{R}$ package (Bivand and Lewin-Koh 2016). The track ends once the trajectory is back at the colony. In practice, we chose to stop the simulation once a location is simulated within a $20-\mathrm{km}$ radius around Kerguelen Island.

\section{RESUlts}

The model described in section Model details was fitted to 15 elephant seal tracks, each corresponding to one individual trip away from the colony. The tracks comprise about 7,300 locations, and it took around one minute to fit the model on a dual-core i5 CPU.

We include the code used to fit the model in the Data S1. Note that, for speed, we implemented the likelihood function in $\mathrm{C}++$, using Rcpp (Eddelbuettel et al. 2011). In Data $\mathrm{S} 1$, we also provide the data set comprising the 15 tracks.

\section{Estimated turn and step-length distributions}

Fig. 2 shows histograms of the step lengths and turning angles of the data, on which are plotted the estimated state-dependent gamma and von Mises densities. The state-dependent densities for each state here have been weighted according to the proportion of time the corresponding state is active, as determined using the Viterbi algorithm (Zucchini et al. 2016). Similarly, for both the step lengths and the turning angles, Fig. 2
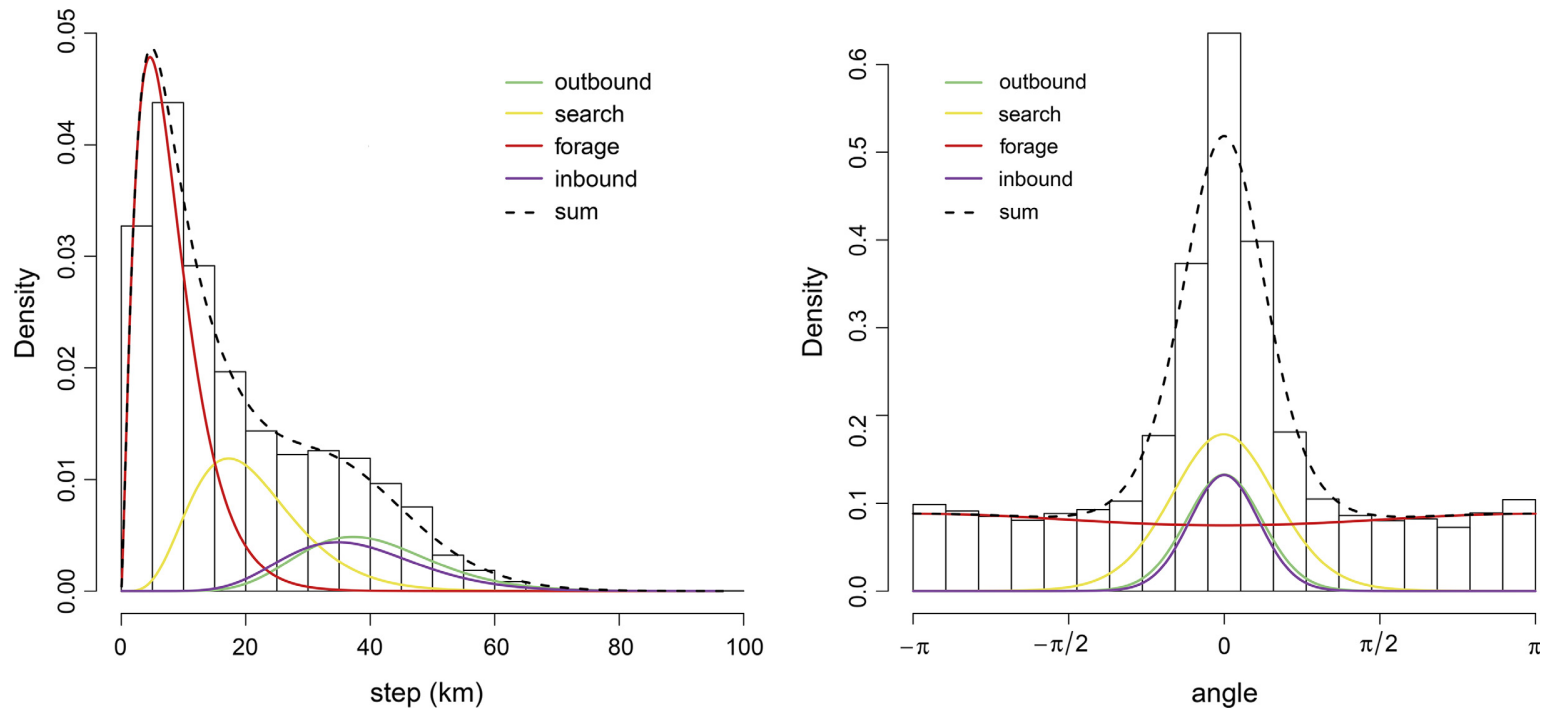

FIG. 2. Estimated state-dependent distributions for the step lengths (left) and the turning angles (right). (For colors, refer to the online version of the paper.) [Color figure can be viewed at wileyonlinelibrary.com] 
also displays the cumulative distribution, i.e., the sum of these weighted densities. Based on visual inspection, these cumulative distributions do not indicate any lack of fit of the model.

Formal model checks can be conducted using forecast pseudo-residuals, which use the probability integral transform to effectively compare the observation at each time $t$ to the associated forecast distribution based on the observations up to time $t-1$. In case of the turning angles, the definition of the pseudo-residuals is essentially arbitrary due to the circular nature of this variable (cf. Langrock et al. 2012). Thus, we restrict the model check to the step length variable. The quantile-quantile plot of the pseudo-residuals for the step lengths, against the standard normal distribution, is shown in Appendix S1 (Section S4). A few of the tracks include steps of slow movement near the colony. They are not captured by the "outbound" and "inbound" states of fast movement, such that they appear as outliers in the qq-plot; this could be resolved during the preprocessing, by excluding the corresponding observations. The model also slightly underestimates the number of long steps (roughly between 40 and $50 \mathrm{~km}$ ). In this regard, the fit could be improved by using more flexible step length distributions, albeit at a computational cost (Langrock et al. 2015). However, note that the improvement in inference on the stateswitching dynamics would be minimal.

The states corresponding to the outbound and inbound movements display very similar features, with high step lengths and strong directional persistence; the distinction is that the colony acts as a centre of repulsion in the former, and a centre of attraction in the latter. The foraging phases are characterized by shorter steps, i.e., slower movement, and less directional persistence, with a roughly flat distribution of turning angles. In the searchtype movement mode, the model captures moderately long steps and directed movement, making it clearly distinct from foraging behaviour.

The estimates of all the model parameters are provided in Appendix S1 (Section S2).

\section{Estimated state sequences}

The most probable state sequence was computed with the Viterbi algorithm. Fig. 3 shows the 15 tracks, coloured by decoded states. The individual decoded tracks are provided in Appendix S1 (Section S1). In all tracks, the first state corresponds to the animal moving quickly towards the south. Then, the behaviour alternates between searching (state 2) and foraging (state 3 ) periods, typically near the ice or in Antarctic continental shelf waters. In general, more northerly search behaviour is apparent in the westernmost tracks. Eventually, the animal switches to state 4 as it starts moving back towards the island colony.

Overall, the model appears to adequately identify the outbound and inbound trips. However, we suspect that the decoded state sequence might sometimes fail to capture the exact timing of the transitions out of the outbound state, and the transitions into the inbound state. In some tracks, the animal goes through a transitional phase, between the outbound trip and search behaviour, in which the movement is slower but still very directed. Although these periods are still arguably part of the outbound trip, they might be attributed to the searching state, due to the decrease in speed. The same situation arises during the transition from search to inbound trip.

Fig. 4 demonstrates state decoding more specifically, on the track presented in Fig. 1. In particular, subFig. 4(C) shows how the localized movements of the elephant seal near the Antarctic continent is split into two very distinct behaviours, which seem to be apportioned adequately between states 2 and 3 .

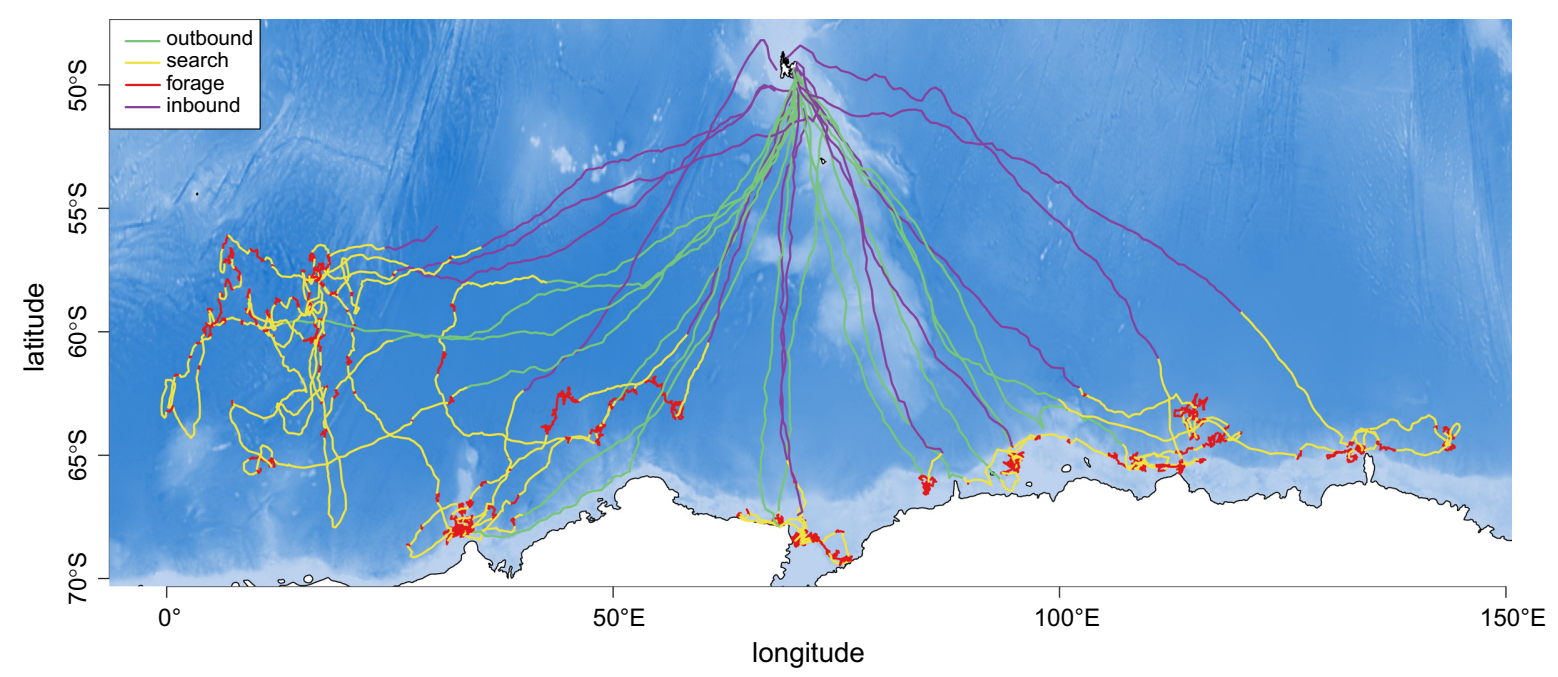

FIG. 3. Fifteen elephant seal tracks, colored by Viterbi-decoded states. The white area at the bottom is the Antarctic continent. (For colors, refer to the online version of the paper.) [Color figure can be viewed at wileyonlinelibrary.com] 
(A)

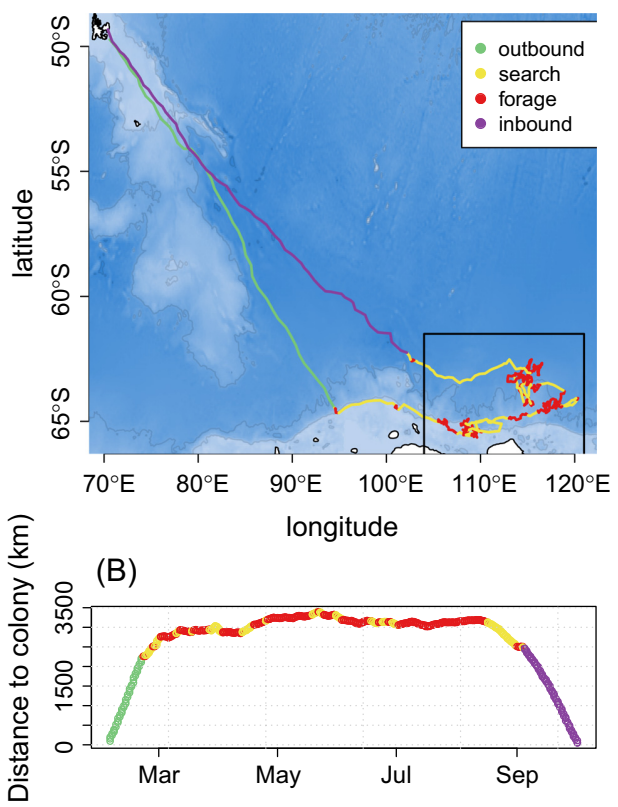

(C)

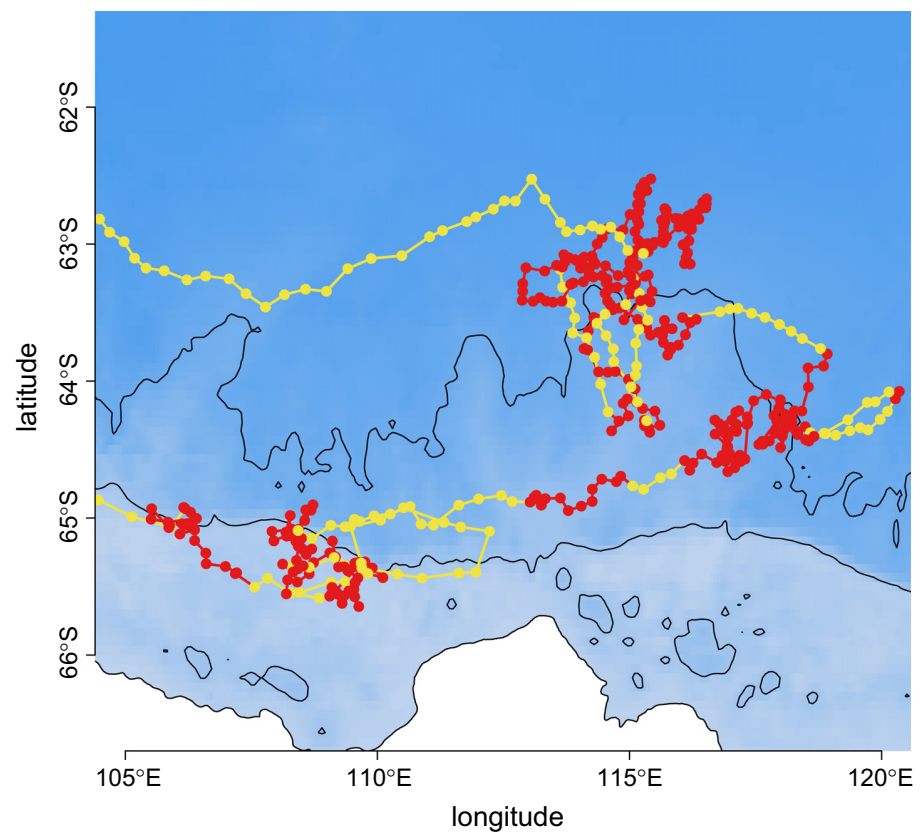

FIg. 4. (A) An example track of a southern elephant seal. (B) The distance from Kerguelen Island through time. (C) A "zoomed in" part of the track shown in (A). The four colors correspond to the most probable states, decoded with the Viterbi algorithm. (A) and (B) clearly illustrate the phases of fast and directed movement, which are attributed to the outbound and inbound trips. (C) shows in more detail the different patterns in the animal's movement, when near the sea ice region, which distinguish between search and foraging behaviours. (For colors, refer to the online version of the paper.) [Color figure can be viewed at wileyonlinelibrary.com]

\section{Estimated effects of covariates}

The transition probabilities were estimated as functions of the distance to the colony, and the time spent away from the colony, as described in section Model details. Fig. 5 displays plots of the transition probabilities from state 1 to state 2 (end of outbound trip), and from state 2 to state 4 (start of inbound trip).

The HMM predicted that elephant seals were unlikely to switch away from state 1 when close to the colony, but the probability increased quickly at distances $>3000 \mathrm{~km}$, when the animals tend to start searching for foraging patches. Moreover, during the first few months away from the colony, elephants seals do not switch to state 4 (return trip), but instead tend to cycle through search and foraging phases. Later, after about 6 months, the probability of switching to state 4 starts to increase.

This is consistent with the annual cycle in this species and the timing of return to the island colony after the long post-moult migration (McCann 1980, Hindell and Burton 1988, Slip and Burton 1999). Therefore, the estimated relationships between covariates and behaviour is consistent with the known behaviour of elephant seals and their annual moulting and breeding cycles.

\section{Simulation results}

One of the main advantages of our approach over simpler HMMs (e.g., HMMs with fewer states, no constraints on the switching probabilities, only based on CRWs. ..) is the possibility to simulate realistic movement tracks from the fitted model. The simulation procedure is described in section Simulation from fitted model. Fig. 6 shows ten tracks, simulated from the fitted model.

The simulated tracks display many of the features of the real ones. To compare them, we simulated 100 tracks, and summarized the proportion of time allocated to each state, and the mean duration spent in each behaviour (before switching to another behaviour), in Table 1. In the simulated data, we find the overall proportional state allocation very well represented. However, we find on average slightly shorter behavioural phases for states 2 and 3 (search and forage) than in the real data. This may partly be due to the assumed Markov property of the state process which, for states not affected by covariates (e.g., the foraging state), implies that the times spent within the state are geometrically distributed (Zucchini et al. 2016). We suggest ways to relax this assumption in section Progress toward prediction from estimated process models. Histograms of the dwell times in each state, for the real and simulated tracks, are provided in Appendix S1 (Section S3). 

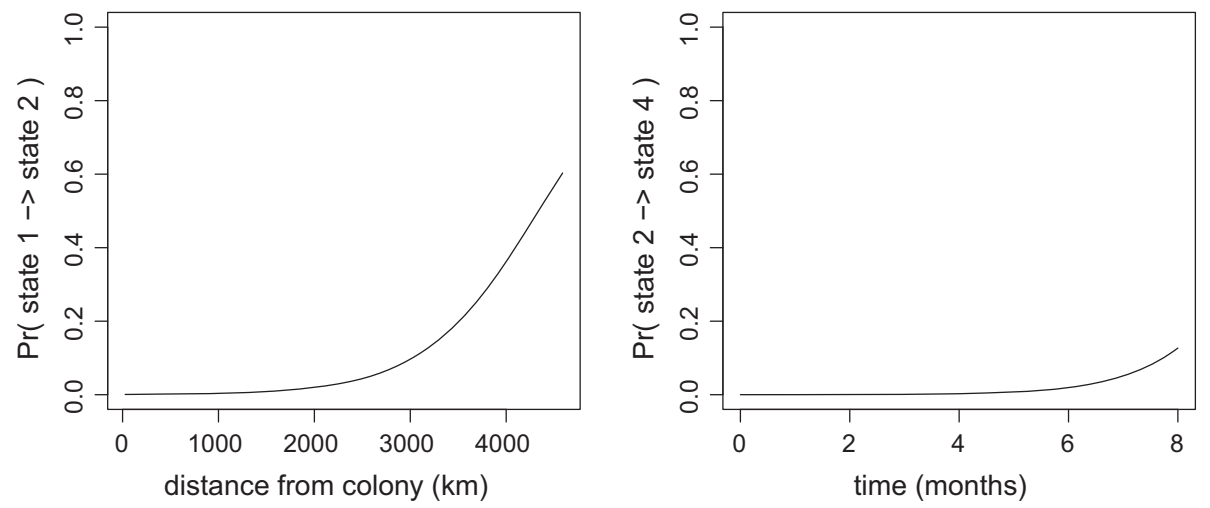

FIG. 5. Transition probabilities from outbound to search as a function of the distance from the colony (left), and from search to inbound as a function of the time spent away from the colony (right).

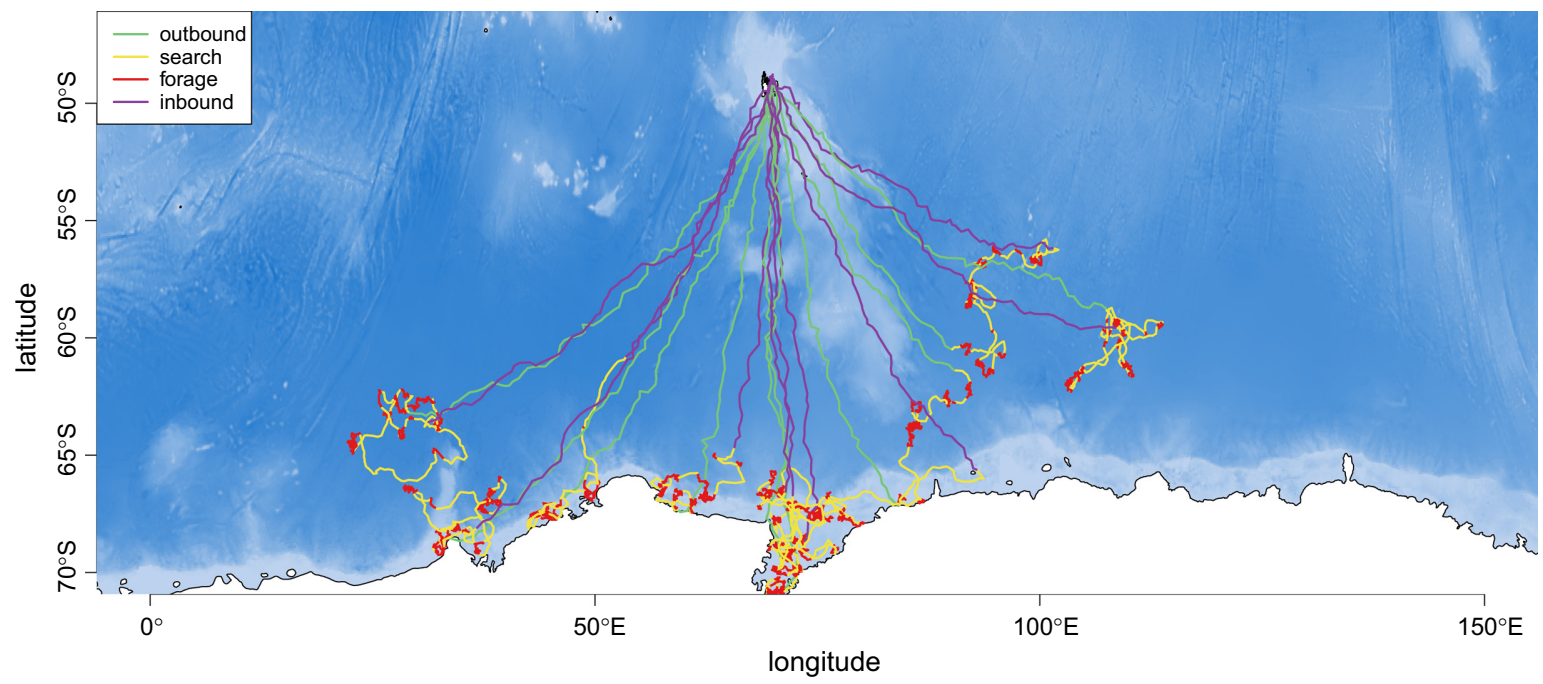

FIG. 6. Ten simulated movement tracks, obtained with the MLE of the parameters of the fitted model. (For colors, refer to the online version of the paper.) [Color figure can be viewed at wileyonlinelibrary.com]

The simulation successfully captures the southward direction of the outbound trip. Then, the process switches to search and forage behaviours at a realistic distance to the colony, as the probability of this transition is a function of the distance to Kerguelen Island. The extent of movements within each state, which is informed by the estimated step and angle distributions, is also realistic. However, the spatial distribution of foraging activity is not tied to correspond to what is observed in real tracks. Thus, the exact locations of searching and foraging activities in the simulated data are of no environmental relevance. This could be improved by including environmental covariates; this is discussed in more detail in section Progress toward prediction from estimated process models.

The durations of simulated trips are reasonable for the study species: out of the 100 simulated trips, 90 lasted between 5 and 9 months.
TABLE 1. Comparison of the real tracks and 100 simulated tracks, in terms of overall proportion of observations attributed to each state, and of mean dwell time in each behaviour (in days).

\begin{tabular}{lccccc}
\hline \hline & \multicolumn{2}{c}{ Overall proportion } & & \multicolumn{2}{c}{ Mean state dwell time } \\
\cline { 2 - 3 } \cline { 5 - 6 } & $\begin{array}{c}\text { Real } \\
\text { data }\end{array}$ & $\begin{array}{c}\text { Simulated } \\
\text { data }\end{array}$ & & $\begin{array}{c}\text { Real } \\
\text { data }\end{array}$ & $\begin{array}{c}\text { Simulated } \\
\text { data }\end{array}$ \\
\hline State 1 & $12.9 \%$ & $11.2 \%$ & & 25.4 & 23.2 \\
State 2 & $24.2 \%$ & $23.3 \%$ & & 4.5 & 3.6 \\
State 3 & $51.1 \%$ & $53.7 \%$ & & 10.4 & 9.0 \\
State 4 & $11.7 \%$ & $11.8 \%$ & & 24.6 & 24.4 \\
\hline
\end{tabular}

\section{Discussion}

We have described a method for modelling the tripbased movements of animals undertaking central place foraging. This approach uses a hidden Markov model to 
directly estimate state movement and switching parameters from empirical telemetry observations. The model handles the natural sequence of behaviours within a trip, i.e., "outbound", "search", "forage", and "inbound".

\section{Comparisons to simpler models}

The four-state HMM we have constructed is relatively complex: it mixes biased and correlated random walks, and the transition probabilities depend on time-varying covariates (distance from colony, and time). It is therefore necessary to consider what we gain from using a complex behavioural model over simpler models. For example, two-state switching CRW models have commonly been used (e.g., Morales et al. 2004, Hindell et al. 2016), and can be easily fitted across a range of datasets, e.g., using the R packages moveHMM (Michelot et al. 2016) or bsam (Jonsen 2016).

One key inference from the more complex model described in this manuscript is the length of outbound and inbound journeys (both in distance and in time). In this trip-based HMM, we can estimate this directly with the most likely state sequence, derived with the Viterbi algorithm. Arguably, one could apply heuristic rules to the state estimates from a two-state model, to obtain the same thing. For example, the outbound trip could be taken to start when the animal leaves the colony, and end when the proportion of observations categorized as "resident" behaviour reaches a threshold $p$ (where $p$ is relatively small, say $p=0.1$ ). This would have the effect of ignoring short runs of resident behaviour within the transit. Similar rules could be envisaged based on distance from the island, for example. These heuristic rules of thumb may be useful, but suffer from a degree of arbitrariness.

Hidden Markov models with more than two states have been used to model fishing vessel trips - which can be considered, most basically, as another top predator. For example, Vermard et al. (2010) and Walker and Bez (2010) used 3-state HMMs to distinguish "fishing", "steaming", and "still" behaviours of fishing vessels. Peel and Good (2011) consider a 5-state HMM, with the addition of states for "entry" and "exit" movement between the latter two behaviours. In such models, simulation from fitted models could be a useful extension. However, to the best of our knowledge, they have not been used for that purpose.

The disadvantage of our trip-based 4-state model is its aforementioned complexity. Without reasonable starting values in the maximum likelihood estimation, the parameter estimation routines can provide poor parameter estimates, by finding local maxima of the likelihood, or fail to converge altogether. These are well-known problems with numerical maximum likelihood, which require careful attention. One way to address this numerical problem is to run the estimation with many different sets of starting values (possibly chosen at random), and compare the resulting estimates. In the case study, we tried 50 sets of randomly chosen starting values in order to ensure that we identified the global maximum of the likelihood function.

\section{Progress toward prediction from estimated process models}

A key feature of the models we have demonstrated is that they are able to generate simulated tracks which capture certain aspects of the behaviour of seals. While it is clear that these are gross simplifications of the true movements of CPF predators, the simulations are nevertheless useful for predicting aggregate properties from the fitted model. For instance, we might predict the average spatial distribution of seals from the fitted model. We can also compute a distribution of arrival and departure times from a given area, which can be useful for assessing effectiveness of spatial management regimes or reserve usage and connectivity between populations (Abecassis et al. 2013, Guan et al. 2013, Kanagaraj et al. 2013, Stehfest et al. 2015).

Currently, these models do not contain detailed environmental or biological predictors, which are known to be important in influencing southern elephant seal behaviour (Pinaud and Weimerskirch 2005, Bestley et al. 2013); for instance, the role of specific oceanographic variables (Biuw et al. 2007, Labrousse et al. 2015). It is in principle straightforward to include additional covariates in the model described in section Model details; though doing so might increase numerical instability. Nonetheless, incorporating such variables will be important if these models are to truly realise their potential for understanding how CPF marine predators might respond to changing environmental regimes. For the case of southern elephant seals, we could for instance express the transition from outbound to search in terms of distance to the sea ice edge, instead of distance from the colony. We could also investigate the apparent state 2 behaviour observed further from the Antarctic continent, which may well be indicative of the animal moving slowly toward the colony and away from the continent as the ice advances northwards. Other variables, such as response to different water masses, frontal zones, etc., are likely to be more subtle, and may serve to influence transitions between search and forage states.

Including environmental covariates to inform the probabilities of switching may also lead to more realistic simulated tracks. In particular, it could help to simulate more realistic state dwell-times. An alternative is to use so-called hidden semi-Markov models (Langrock et al. 2012), where the geometric state dwell-time distribution can be replaced by more flexible distributions. In simulations, additional covariates would also help to inform the spatial distribution of foraging activities.

The approach presented here demonstrates progress toward melding telemetry and sensor data with spatially explicit prediction of animal distributions and behaviour. Hidden Markov and state space models have much to offer in this prospect (Patterson et al. 2008), 
but have thus far been rather limited in being used for the purposes of prediction. A long-term goal for animal movement research is the general prediction of realistic movements modelled (i.e., statistically estimated from a process model) from empirical data collected at the individual level, but applied to novel situations and scaled up to population-level responses. These might for example include projections of future environmental conditions (Perry et al. 2005, Trathan et al. 2007, Hazen et al. 2013), or application to changed colony conditions.

For the ultimate goal of building empirically and mechanistically based simulation models to be realized, we believe that it is necessary to directly estimate process models which capture the key aspects of animal biology sufficiently well. Recent studies have demonstrated that highly complex simulation models incorporating physiological details, habitat information, etc., can be built (Schick et al. 2013, New et al. 2014). However, typically such models are either data limited or unable to be directly estimated from empirical observations. As such, it is likely that there will be limitations to the degree of complexity which can be realised in estimated models. The consequence of this is that attempting to cleanly move from an estimated model to a simulation and prediction exercise will encounter difficulties as the estimation model fails to capture certain fundamental aspects of the real movements. A simple example of this is the behaviour of marine animals in regard to coastlines. Arbitrary, but probably reasonable measures, such as using a rejection step to restrict animals to remain in the ocean are necessary to mimic the straightforward reality that marine animals do not typically wander over land masses. Despite the apparent triviality of this point, it is informative to consider, as it highlights elements needed at the simulation and prediction phase, but which may not fit within an estimation model.

\section{Concluding remarks}

Building on the general framework of Markov-switching random walks and hidden Markov models, our method accommodates naturally trip-based movement of central place foragers. It offers a fast way to categorize movement tracks into behavioural modes, and to describe the underlying mechanics of behavioural switching in terms of time-varying covariates. We believe that models like those presented here begin to address the interesting three way trade-off between (1) complexity and realism, (2) the desirable aspects of direct estimation using rigorous statistical inference, and (3) computational efficiency. The first aspect allows simulations to capture many features of the real data and makes the models potentially useful for prediction at the individual level. The second brings the power and objectivity of statistical methods as a way to understand the spatial dynamics of animals. The final point allows for ease of use, and means that more realistic models can be applied to large data sets.

\section{ACKNOWLEDGMENTS}

TM and TP received support from IMBER-CLIOTOP and Macquarie University Safety Net Grant 9201401743. SB was supported under an Australia Research Council Super Science Fellowship. IDJ was supported by a Macquarie Vice-Chancellors Innovation Fellowship. TAP was supported by a CSIRO Julius Career Award and the Villum Foundation. The southern elephant seal data was sourced from the Integrated Marine Observing System (IMOS). The tagging program received logistics support from the Australian Antarctic Division and the French Polar Institute (Institut Paul-Émile Victor, IPEV). All tagging procedures were approved and carried out under the guidelines of the University of Tasmania Animal Ethics Committee and the Australian Antarctic Animal Ethics Committee. Initial ideas from Mark Bravington, Uffe Thygesen and Martin Pedersen were very helpful in formulating earlier versions of these models. We thank an anonymous reviewer for helpful feedback on an earlier version of the manuscript.

\section{Literature Cited}

Abecassis, M., I. Senina, P. Lehodey, P. Gaspar, D. Parker, G. Balazs, and J. Polovina. 2013. A model of loggerhead sea turtle (Caretta caretta) habitat and movement in the oceanic north pacific. PLoS ONE 8:e73274.

Ashmole, N. P. 1963. The regulation of numbers of tropical oceanic birds. Ibis 103:458-473.

Auger-Méthé, M., A. E. Derocher, C. A. DeMars, M. J. Plank, E. A. Codling, and M. A. Lewis. 2016. Evaluating random search strategies in three mammals from distinct feeding guilds. Journal of Animal Ecology 85:1411-1421.

Bell, W. J. 1990. Central place foraging. Pages 171-187 in Searching behaviour. Springer, Dordrecht, The Netherlands.

Bestley, S., I. D. Jonsen, M. A. Hindell, C. Guinet, and J.-B. Charrassin. 2013. Integrative modelling of animal movement: incorporating in situ habitat and behavioural information for a migratory marine predator. Proceedings of the Royal Society of London B: Biological Sciences 280: 20122262.

Biuw, M., et al. 2007. Variations in behavior and condition of a southern ocean top predator in relation to in situ oceanographic conditions. Proceedings of the National Academy of Sciences 104:13705-13710.

Bivand, R., and N. Lewin-Koh. 2016. maptools: Tools for Reading and Handling Spatial Objects. https://CRAN.R-project. org/package $=$ maptools. $\mathrm{R}$ package version 0.8-39.

Boyd, C., A. E. Punt, H. Weimerskirch, and S. Bertrand. 2014. Movement models provide insights into variation in the foraging effort of central place foragers. Ecological Modelling 286:13-25.

Breed, G. A., I. D. Jonsen, R. A. Myers, W. D. Bowen, and M. L. Leonard. 2009. Sex-specific, seasonal foraging tactics of adult grey seals (Halichoerus grypus) revealed by statespace analysis. Ecology 90:3209-3221.

Breed, G. A., E. A. Golson, and M. T. Tinker. 2016. Predicting animal home-range structure and transitions using a multistate ornstein-uhlenbeck biased random walk. Ecology 98:32-47.

Carrick, R., S. Csordas, S. E. Ingham, and K. Keith. 1962. Studies on the southern elephant seal, Mirounga leonina (L.). iii. the annual cycle in relation to age and sex. Wildlife Research 7:119-160.

Codling, E., M. Plank, and S. Benhamou. 2008. Random walk models in biology. Journal of the Royal Society Interface 5:813-834. 
Croxall, J. P., S. H. Butchart, B. Lascelles, A. J. Stattersfield, B. Sullivan, A. Symes, and P. Taylor. 2012. Seabird conservation status, threats and priority actions: a global assessment. Bird Conservation International 22:1-34

DeRuiter, S. L., R. Langrock, T. Skirbutas, J. A. Goldbogen, J. Calambokidis, A. S. Friedlaender, and B. L. Southall. 2017. A multivariate mixed hidden Markov model for blue whale behaviour and responses to sound exposure. Annals of Applied Statistics 11:362-392.

Eddelbuettel, D., R. François, J. Allaire, J. Chambers, D. Bates, and K. Ushey. 2011. Rcpp: seamless R and C++ integration. Journal of Statistical Software 40:1-18.

Fauchald, P., and T. Tveraa. 2003. Using first-passage time in the analysis of area-restricted search and habitat selection. Ecology 84:282-288.

Fauchald, P., and T. Tveraa. 2006. Hierarchical patch dynamics and animal movement pattern. Oecologia 149:383395.

Guan, W., J. Cao, Y. Chen, and M. Cieri. 2013. Impacts of population and fishery spatial structures on fishery stock assessment. Canadian Journal of Fisheries and Aquatic Sciences 70:1178-1189.

Hamer, D., S. Goldsworthy, D. Costa, S. Fowler, B. Page, and M. Sumner. 2013. The endangered Australian sea lion extensively overlaps with and regularly becomes by-catch in demersal shark gill-nets in South Australian shelf waters. Biological Conservation 157:386-400.

Hays, G. C., et al. 2016. Key questions in marine megafauna movement ecology. Trends in Ecology and Evolution 31: 463-475.

Hazen, E. L., et al. 2013. Predicted habitat shifts of pacific top predators in a changing climate. Nature Climate Change 3:234-238.

Higginson, A. D., and A. I. Houston. 2015. The inuence of the food-predation trade-off on the foraging behaviour of central-place foragers. Behavioral Ecology and Sociobiology 69:551-561.

Hijmans, R. J. 2016. geosphere: Spherical Trigonometry. https:// CRAN.R-project.org/package $=$ geosphere. $\mathrm{R}$ package version 1.5-5.

Hindell, M. A., and H. R. Burton. 1988. Seasonal haul-out patterns of the southern elephant seal (Mirounga leonina L.), at Macquarie Island. Journal of Mammalogy 69:81-88.

Hindell, M. A., et al. 2016. Circumpolar habitat use in the southern elephant seal: implications for foraging success and population trajectories. Ecosphere 7:e01213.

Jabour, J., M.-A. Lea, S. D. Goldsworthy, G. Melcher, K. Sykes, and M. A. Hindell. 2016. Marine telemetry and the conservation and management of risk to seal species in Canada and Australia. Ocean Development and International Law 47: 255-271.

Jonsen, I. 2016. Joint estimation over multiple individuals improves behavioural state inference from animal movement data. Scientific Reports 6:20625.

Jonsen, I. D., J. M. Flemming, and R. A. Myers. 2005. Robust state-space modeling of animal movement data. Ecology $86: 2874-2880$.

Jonsen, I., M. Basson, S. Bestley, M. Bravington, T. Patterson, M. W. Pedersen, R. Thomson, U. H. Thygesen, and S. Wotherspoon. 2013. State-space models for bio-loggers: a methodological road map. Deep Sea Research Part II: Topical Studies in Oceanography 88:34-46.

Kanagaraj, R., T. Wiegand, S. Kramer-Schadt, and S. P. Goyal. 2013. Using individual-based movement models to assess inter-patch connectivity for large carnivores in fragmented landscapes. Biological Conservation 167:298-309.
Kareiva, P., and G. Odell. 1987. Swarms of predators exhibit "prey taxis" if individual predators use area-restricted search. American Naturalist 130:233-270.

Kristensen, K., A. Nielsen, C. W. Berg, H. Skaug, and B. M. Bell. 2016. TMB: Automatic differentiation and Laplace approximation. Journal of Statistical Software 70:1-21.

Labrousse, S., et al. 2015. Winter use of sea ice and ocean water mass habitat by southern elephant seals: The length and breadth of the mystery. Progress in Oceanography 137:52-68.

Langrock, R., R. King, J. Matthiopoulos, L. Thomas, D. Fortin, and J. M. Morales. 2012. Flexible and practical modeling of animal telemetry data: hidden Markov models and extensions. Ecology 93:2336-2342.

Langrock, R., J. G. C. Hopcraft, P. G. Blackwell, V. Goodall, R. King, M. Niu, T. A. Patterson, M. W. Pedersen, A. Skarin, and R. S. Schick. 2014. Modelling group dynamic animal movement. Methods in Ecology and Evolution 5:190-199.

Langrock, R., T. Kneib, A. Sohn, and S. L. DeRuiter. 2015. Nonparametric inference in hidden Markov models using P-splines. Biometrics 71:520-528.

Leos-Barajas, V., T. Photopoulou, R. Langrock, T. A. Patterson, Y. Watanabe, M. Murgatroyd, and Y. P. Papastamatiou. 2017. Analysis of animal accelerometer data using hidden Markov models. Methods in Ecology and Evolution 8:161-173.

Lonergan, M., C. Duck, D. Thompson, B. Mackey, L. Cunningham, and I. Boyd. 2007. Using sparse survey data to investigate the declining abundance of British harbour seals. Journal of Zoology 271:261-269.

Martin, G. R., and R. Crawford. 2015. Reducing bycatch in gillnets: a sensory ecology perspective. Global Ecology and Conservation 3:28-50.

Matthiopoulos, J. 2003. Model-supervised kernel smoothing for the estimation of spatial usage. Oikos 102:367-377.

McCann, T. 1980. Population structure and social organization of southern elephant seals, Mirounga leonina (L.). Biological Journal of the Linnean Society 14:133-150.

McClintock, B. T., R. King, L. Thomas, J. Matthiopoulos, B. J. McConnell, and J. M. Morales. 2012. A general discrete-time modeling framework for animal movement using multistate random walks. Ecological Monographs 82:335-349.

McKellar, A. E., R. Langrock, J. R. Walters, and D. C. Kesler. 2015. Using mixed hidden Markov models to examine behavioral states in a cooperatively breeding bird. Behavioral Ecology $26: 148-157$.

Michelot, T., R. Langrock, and T. A. Patterson. 2016. moveHMM: an $\mathrm{R}$ package for the statistical modelling of animal movement data using hidden Markov models. Methods in Ecology and Evolution 7:1308-1315.

Morales, J. M., D. T. Haydon, J. Frair, K. E. Holsinger, and J. M. Fryxell. 2004. Extracting more out of relocation data: building movement models as mixtures of random walks. Ecology 85:2436-2445.

New, L. F., et al. 2014. Using short-term measures of behaviour to estimate long-term fitness of southern elephant seals. Marine Ecology Progress Series 496:99-108.

Olsson, O., and A. Bolin. 2014. A model for habitat selection and species distribution derived from central place foraging theory. Oecologia 175:537-548.

Oppel, S., et al. 2015. Foraging distribution of a tropical seabird supports ashmole's hypothesis of population regulation. Behavioral Ecology and Sociobiology 69:915-926.

Pante, E., and B. Simon-Bouhet. 2013. marmap: a package for importing, plotting and analyzing bathymetric and topographic data in R. PLoS ONE 8:e73051.

Patrick, S. C., S. Bearhop, D. Grémillet, A. Lescroël, W. J. Grecian, T. W. Bodey, K. C. Hamer, E. Wakefield, M. Le 
Nuz, and S. C. Votier. 2014. Individual differences in searching behaviour and spatial foraging consistency in a central place marine predator. Oikos 123:33-40.

Patterson, T. A., L. Thomas, C. Wilcox, O. Ovaskainen, and J. Matthiopoulos. 2008. State-space models of individual animal movement. Trends in Ecology and Evolution 23:87-94.

Patterson, T. A., A. Parton, R. Langrock, P. G. Blackwell, L. Thomas and R. King. 2016. Statistical modelling of animal movement: a myopic review and a discussion of good practice. arXiv 1603.07511.

Peel, D., and N. M. Good. 2011. A hidden Markov model approach for determining vessel activity from vessel monitoring system data. Canadian Journal of Fisheries and Aquatic Sciences 68:1252-1264.

Perry, A. L., P. J. Low, J. R. Ellis, and J. D. Reynolds. 2005. Climate change and distribution shifts in marine fishes. Science 308:1912-1915.

Photopoulou, T., M. A. Fedak, J. Matthiopoulos, B. McConnell, and P. Lovell. 2015. The generalized data management and collection protocol for conductivity-temperature-depth satellite relay data loggers. Animal Biotelemetry 3:1.

Pinaud, D., and H. Weimerskirch. 2005. Scale-dependent habitat use in a long-ranging central place predator. Journal of Animal Ecology 74:852-863.

Raymond, B., et al. 2015. Important marine habitat off east Antarctica revealed by two decades of multi-species predator tracking. Ecography 38:121-129.

Russell, D. J., B. McConnell, D. Thompson, C. Duck, C. Morris, J. Harwood, and J. Matthiopoulos. 2013. Uncovering the links between foraging and breeding regions in a highly mobile mammal. Journal of Applied Ecology 50:499-509.

Schick, R. S., et al. 2013. Estimating resource acquisition and at-sea body condition of a marine predator. Journal of Animal Ecology 82:1300-1315.
Slip, D. J., and H. R. Burton. 1999. Population status and seasonal haulout patterns of the southern elephant seal (Mirounga leonina) at heard island. Antarctic Science 11:38-47.

Staniland, I., I. Boyd, and K. Reid. 2007. An energy-distance trade-off in a central-place forager, the Antarctic fur seal (Arctocephalus gazella). Marine Biology 152:233-241.

Stehfest, K. M., T. A. Patterson, A. Barnett, and J. M. Semmens. 2015. Markov models and network analysis reveal sex-specific differences in the space-use of a coastal apex predator. Oikos 124:307-318.

Towner, A. V., V. Leos-Barajas, R. Langrock, R. S. Schick, M. J. Smale, T. Kaschke, O. J. Jewell, and Y. P. Papastamatiou. 2016. Sex-specific and individual preferences for hunting strategies in white sharks. Functional Ecology 30:1397-1407.

Trathan, P., J. Forcada, and E. Murphy. 2007. Environmental forcing and southern ocean marine predator populations: effects of climate change and variability. Philosophical Transactions of the Royal Society of London B: Biological Sciences 362:2351-2365.

Vermard, Y., E. Rivot, S. Mahévas, P. Marchal, and D. Gascuel. 2010. Identifying fishing trip behaviour and estimating fishing effort from VMS data using Bayesian hidden Markov models. Ecological Modelling 221:1757-1769.

Wakefield, E. D., R. A. Phillips, J. Matthiopoulos, A. Fukuda, H. Higuchi, G. J. Marshall, and P. N. Trathan. 2009. Wind field and sex constrain the flight speeds of central-place foraging albatrosses. Ecological Monographs 79:663-679.

Walker, E., and N. Bez. 2010. A pioneer validation of a statespace model of vessel trajectories (VMS) with observers data. Ecological Modelling 221:2008-2017.

Zucchini, W., I. L. MacDonald, and R. Langrock. 2016. Hidden Markov models for time series: an introduction using R. Second edition. Chapman and Hall/CRC, Boca Raton, Florida, USA.

\section{SUPPORTING INFORMATION}

Additional supporting information may be found in the online version of this article at http://onlinelibrary.wiley.com/doi/ 10.1002/ecy.1880/suppinfo 